\title{
Pitfalls of ascertainment biases in genome annotations - Computing comparable protein domain distributions in Eukarya
}

\author{
Arli A. Parikesit ${ }^{1-3,9}$, Lydia Steiner ${ }^{1,2}$, Peter F. Stadler ${ }^{2-8}$ and Sonja J. Prohaska ${ }^{1,2 *}$ \\ ${ }^{1}$ Computational EvoDevo Group, Department of Computer Science, University of Leipzig,Härtelstraße 16-18, D-04107 Leipzig, Germany \\ ${ }^{2}$ Interdisciplinary Center for Bioinformatics, University of Leipzig, Härtelstraße 16-18, D-04107 Leipzig, Germany; E-Mail: studla@bioinf.uni-leipzig.de \\ ${ }^{3}$ Max Planck Institute for Mathematics in the Sciences, Inselstrasse 22, D-04103 Leipzig, Germany \\ ${ }^{4}$ Bioinformatics Group, $\quad$ Department of Computer Science, University of Leipzig, Härtelstraße 16-18, D-04107 Leipzig, Germany \\ ${ }^{5}$ Fraunhofer Institut fürZelltherapie und Immunologie-IZI Perlickstraße 1, D-04103 Leipzig,Germany \\ ${ }^{6}$ Department of Theoretical Chemistry, University of Vienna, Währingerstraße 17, A-1090 Wien,Austria \\ ${ }^{7}$ Center for non-coding RNA in Technology and Health, University of Copenhagen, Grønnegårdsvej 3,DK-1870 Frederiksberg C, Denmark \\ ${ }^{8}$ Santa Fe Institute, 1399 Hyde Park Rd., Santa Fe, NM 87501, USA \\ ${ }^{9}$ Bioinformatics Group, Department of Chemistry, Faculty of Mathematics and Science, University of Indonesia \\ *Corresponding Author: arli,lydia,sonja@bioinf.uni-leipzig.de (Arli A. Parikesit)
}

\section{Article history :}

Received 1 February 2013

Revised 24 July 2013

Accepted 27 July 2013

Available online 15 August 2013

GRAPHICAL ABSTRACT

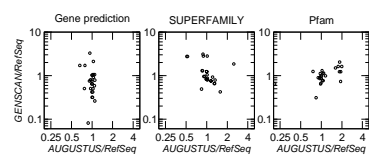

\begin{abstract}
Most investigations into the large-scale patterns of protein evolution are based on gene annotations that have been compiled in reference databases. The use of these resources for quantitative comparisons, however, is complicated by sometimes vast differences in coverage. More importantly, however, we also observe substantial ascertainment biases that cannot be removed by simple normalization procedures. A striking example is provided by the correlations between protein domains. We observe that statistics derived from different computational gene annotation procedure show dramatic discrepancies, and even qualitative changes from negative to positive correlation, when compared to statistics obtained from annotation databases.
\end{abstract}

Keywords: protein domains, HMM models, GO classification, functional genome

(C) 2014 Penerbit UTM Press. All rights reserved http://dx.doi.org/10.11113/mjfas.v10n2.57

\section{INTRODUCTION}

Most proteins are composed of smaller building blocks. These protein domains typically form compact three-dimensional structures that are stable and foldable on their own. Domains convey a specific molecular function such as a particular catalytic activity or binding specificity. Protein function can indeed be inferred from domain content [1]. Protein domains are not only functional units but also constitute fundamental building blocks for protein evolution. They can be readily recombined in different arrangements leading to proteins that utilize different combinations of the same (types of) molecular interactions to fulfill different higher-level functions [2-4]. Over very large evolutionarily time scales, such as those of interest in a comparative analysis of the eukaryotic kingdom, protein domainsare rearranged by fusions, fissions, and terminal loss so that larger proteins are a composite of domains deriving from several ancestral sources [5,6]. The reshuffling of domains is indeed much more frequent than the innovation of novel protein domains $[7,8]$.

It becomes impossible at large time-scales to identify orthologous proteins [9]. One reason is that sequence similarity can degrade beyond recognition. More fundamentally, however, the re-shuffling of protein domains breaks the very concept of orthology as novel proteins arise through fusion and fission from multiple ancestors. The abundance and co-occurrence of protein domains thus becomes the most natural and promising framework to understand patterns of protein evolution, see e.g. [10-12]. In [8], for instance, it is shown that frequent gains and losses of domains lead to significant differences in functional profiles of major eukaryotic clades. Their results argue for a complex last eukaryotic common ancestor and reveal that animals are gaining increased regulatory complexity at the expense of their metabolic capabilities. Similarly, the rise of chromatin-based regulation mechanisms in crown-group eukaryotes can be traced by considering abundances and co-occurrences of the relevant protein domains [11]. A growing core of combinations in multicellular organisms was demonstrated by network analysis of domain co-occurrences [13]. The fundamental role of domains is also emphasized by the emergence of "supra-repeats", i.e., complex and multidomain repetitive patterns, which is of importance e.g. in nucleic acid-binding and protein-protein interaction and hence plays a key role in gene regulation [14]. The diversification of such regulatory proteins, thus translates primarily into statistically unexpected co-occurences of 
protein domains of the same functional type or structural family. A case in point is e.g. the rapid expansion of $\mathrm{KRAB} /$ zinc finger transcription factors in primates [15]. Protein domains are characterized by local amino-acid patterns and hence can be annotated computationally in protein sequences. Several databases, most notably the SUPERFAMILY [16] compiles domain annotations for the known and predicted proteins of a wide variety of species. As we shall demonstrate in this contribution, quantitative comparisons between distant species one the basis of this data, however, are plagued by large differences and biases in coverage. In principle, the most complete information about the protein complement can be inferred from the genome sequence. We therefore suggest a workflow centered around de novo gene predictions to obtain quantitatively comparable estimates, see Fig. 1. We observe, however, that different gene predictors still lead to qualitatively different results.

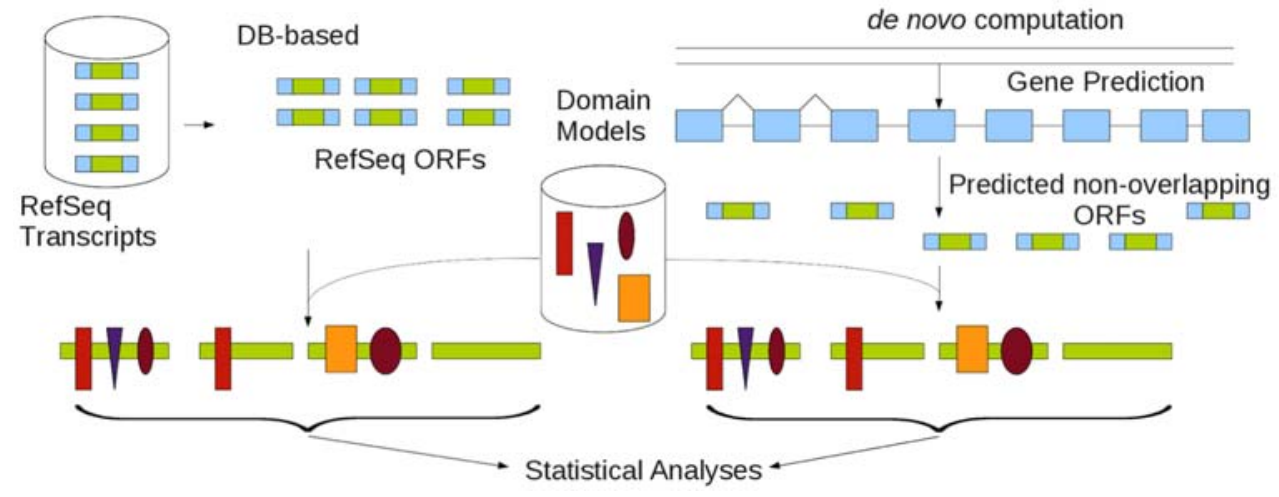

Fig. 1 Work flows for the estimation of domain abundance data from annotation data (1.h.s.) and starting with a de novo gene annotation (r.h.s). In both scenarios, protein domains compiled in databases such as Pfam or SUPERFAMILY are mapped to the known or predicted proteins and form the basis for subsequent statistical analysis.

This contribution is organized as follows: In the following section we briefly outline the methods and data employed for gene prediction, protein domain annotation, and statistical evaluation. In section 3.1 we discuss differences in coverage and outline other potential sources of biases. We then proceed to show that the discrepancies between curated annotation and two distinct gene prediction tools can be large and vary substantially between genomes. In section 3.4 we investigate correlations of functionally defined classes of domains. Here the various sources of biases conspire to produce even qualitatively different results. We finally discuss the resulting limitations of the accuracy of the domain distribution data and the reliability of conclusions drawn from them.

\section{EXPERIMENTAL}

\section{$2.1 \quad$ Sequence data}

We consider the following 18 species with sequenced genomes covering the entire phylogenetic range of the eukaryotes: H.sa: Homo sapiens hg19, D.me: Drosophila melanogaster BDGP5.13, C.el: Caenorhabditis elegans WS200, S.po:Schizosaccharomyces pombe EF1, A.ni: Aspergillus niger CADRE, D.di: Arabidopsis thaliana TAIR9.55, C.re: Clamydomonas Chlre4, T.th: Tetrahymena thermophila tta1 oct2008, P.fa: Plasmodium falciparum PlasmoDB-7.0, L.ma: Leishmania major Lmj 20070731 V5.2, G.la: Giardia lamblia WBC6, T.va:
Trichomonas vaginalis TrichDB-1.2, T.br: Trypanosoma brucei Tb927 May08 v4, N.gr: Naegleria gruberi Naegr1, T.ps: Thalassiosira pseudonana THAPS P.ra: Phytophthora ramorum Phyra1 1, O.sa: Oryza sativa OSV6.1, D.di: Dictyostelium discoideum DDB. Sources are listed in the Supplement http://www.bioinf.unileipzig.de/supplements/ 12-007.

\subsection{Gene prediction}

Gene prediction tools come in two flavors: homology-based and de novo approaches. Since homologybased methods necessarily transfer any biases from the source annotation to the newly annotated target, only de novo methods are applicable for our purposes. A software package that was widely used in early genome projects is GENSCAN $[17,18]$. It is mostly geared towards vertebrate genomic sequences.

Despite the shortcomings of such a "first generation" gene predictor, good performance has been observed in the literature for a diverse set of only distantly related organism (teleost, nematodes, amphioxus, and fungi) without the need for specific training [19] and it has hence served as the most popular tool until a few years ago [20]. Furthermore, it is much less demanding computational resources. Assuming that is samples in an unbiased manner, a moderate loss in sensitivity would be acceptable for the task at hand: after all we are more interested here in statistical associations than a complete annotation. Moreover, GENSCAN has been used 
extensively in the ENSMBL genome database project [21] and the fugu genome annotations are still largely based on this tool [22].

Following [23] we split long chromosomes into overlapping fragments of about $500 \mathrm{~kb}$ to accommodate the tool's restriction on input length. Protein sequences were extracted directly from the GENSCAN predictions. Duplicate predictions in the overlaps between fragments were removed. A potential shortcoming is that the prediction accuracy may vary substantially with differences in gene architecture.

We therefore employed a trainable gene prediction as an alternative. In this class of tools, the statistical model is trained with a set of known genes from the organism that is to be annotated. We chose AUGUSTUS [24-26] as a representative of trainable tools because it has gained popularity in recent genome annotation projects. Both "Specific" (local) and "Default" (web-based) trained models are used here. We used the tools as described in the AUGUSTUS tutorial [27]. Where available, we made use of the default training sets provided at the AUGUSTUS website. For the remaining species, we used the cDNAs available in GenBank. Redundancies were removed with a dedicated pearl script. The FASTA sequences and their headers were cleaned from meta-characters and gaps. Models were trained in "Specific" mode with the pipeline downloaded from the AUGUSTUS website. For our applications, AUGUSTUS was configured to generate only non-overlapping protein-coding genes. The predicted protein sequences are part of the AUGUSTUS output. We verified with bed-tools that no overlapping sequences were contained in the output [28].

The results of the AUGUSTUS and GENSCAN predictions are compiled in Table 1 and compared with the RefSeq (release 53) genes for each of the 18 species. In order to compare the two training modes of AUGUSTUS with each other and with the RefSeq annotation we computed their overlaps with bed-tools and used lucid chart to compute Venn diagrams so that the displayed overlaps in Fig. 2 are drawn to scale [30].

\subsection{Domain annotation}

We used the entire Pfam version 26.0 database, comprising 33672 domain models as well as the entire collection of 9821 Hidden Markov Models (HMMs) provided by the SUPERFAMILY database (version1.75). In both cases we used HMMER3.0rc1 [31] with an E-value threshold of $\mathrm{E} \leq 10^{-3}$ to map the HMMs to the predicted amino acid sequences as well as the RefSeq proteins.

In order to test the quality of gene predictions we compared the sub-collections of protein sequences with at least one mapped Pfam domain between the gene prediction methods and RefSeq database. A representative selection of these results is shown in Fig. 2. Overall, the specific-trained AUGUSTUS predictions have the best coverage of the manually curated RefSeq and are hence used as data basis for subsequent quantitative analysis.

\section{$2.4 \quad$ Functional classification}

The domain databases contain thousands of distinct domain models. Few domains thus appear with sufficient frequency in a single genome to allow a quantitative statistical analysis. Thus we pooled the occurrence data by larger functional categories. The SUPERFAMILY database offers a "Structural Domain Functional Ontology" providing functional and phenotypic annotations of protein domains at the superfamily and family levels [16]. The Pfam annotation is already integrated into GO database, providing a mapping from Pfam domains to GO ontology terms $[32,33]$.

As example we use here the same high-level functional categories as in previous work [23].

bN binding of nucleic acids: GO:0003676 at superfamily level.

bP binding of proteins with potential nuclear localization: GO:0005515 superfamily level.

rC regulation of chromatin GO:0016568 at superfamily level.

rB regulation of binding: GO:0051098 at superfamily level.

$\mathrm{rE}$ regulators of enzymatic activity:GO:0050790 at superfamily level.

$\mathrm{mS}$ metabolism of saccharides: GO:0005976 at superfamily level.

The four functional groups $\mathrm{bN}, \mathrm{bP}, \mathrm{rC}$, and $\mathrm{rB}$ encapsulate major modes of regulation. Both $\mathrm{bN}$ and $\mathrm{bP}$ play an important role for gene regulation by transcription factors and are among the most abundant GO classes, while $\mathrm{rC}$ focuses on chromatin-based epigenetic regulation.

We have shown in [23] that rCgroup correlates well with the hand-picked collection of domain models that can act as readers, writers, and erasers of histone modification [11]. The domain groups $\mathrm{rE}$ and $\mathrm{mS}$ were intended as a form of controls that a priori we did not expect to correlate in a particular way with either nucleic acid or protein binding domains (bN, bP).

From the co-occurrences of domains in predicted proteins and the map of domains to functional (GO-) classes it is straightforward to obtain the number $n(C, D)$ of cooccurrences of the $C$ and $D$ functional classes. As in [23] we correct $n(C, D)$ for the fact that the same domain $x$ can be a member of both Cand D by counting these cases with a weight of $1 / 2$.

\subsection{Co-occurrence analysis}

For each of the 18 species, we separately evaluated the number of domain co-occurrences and the number of genes in which the domain $x$ and $y$ co-occur. Here $x$ and $y$ can be either individual domains, sets of domains belonging to the same superfamily, or the collections of domains compiled into functional classes according to their GO annotations. Denote by $n_{x}$ the total number of annotated domains belonging to group $x$. The simple estestimate for 
the expected number of domain co-occurrences is $E(x, y)=$ $n_{x} n_{y} / n_{g}$, where $n_{g}$ is the number genes of the genome under consideration. As discussed in [23] this estimate does not account for biases arising from the non-uniform distribution of domains over genes. Let $n_{d}(i)$ be the number of domains predicted for protein $i$, and let $n_{d}=\sum_{i} n_{d}(i)$ be the total number of domain. Then the number of $\mathrm{x}$-domains that occur in genes that also contain a y-domain can be estimated as

$$
E(x \mid y)=\left(n_{x} / n_{d}\right) \sum_{i: y \in i}(n d(i)-1)
$$

where the sum runs over all genes $i$ that contain a domain belonging to group $y$. We obtain an alternative estimate by exchanging $x$ and $y$ in equ.(1).

Table 1 Summary of gene and domain annotations. The left part of the table summarized the results of the gene predictors. For the species with the equal result of both 'Default' and 'Specific' training methods, the entries are shown only once. In the right part of the table, we list the number of genes that contain at least one recognizable protein domain from the SUPERFAMILY and Pfam collections, respectively. The phylogenetic distribution [29] of the 18 investigated species is shown on the left margin.

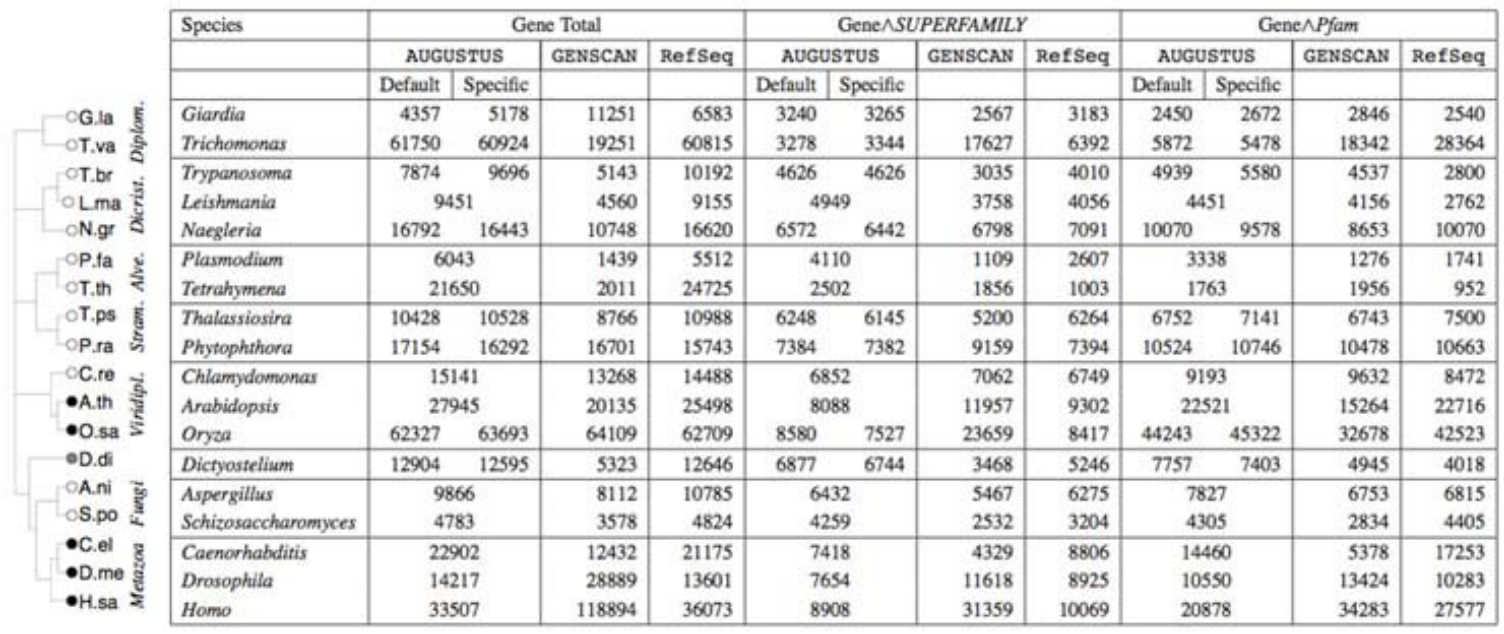

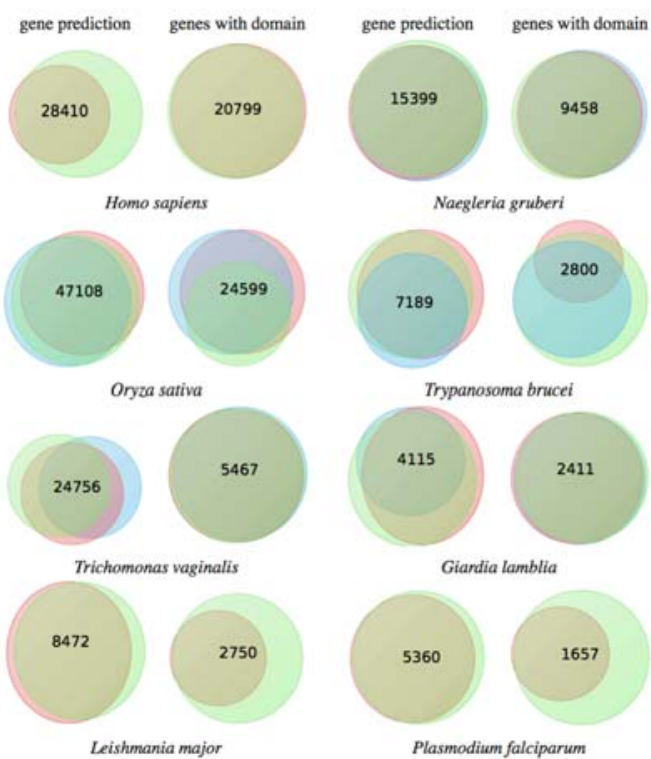

Fig. 2 Comparison of gene predictions for 8 of the 18 species. (See online supplement for additional data). For each species we show a Venn diagram, drawn to scale, for both the raw output of the gene predictions and for the subset of proteins with at least one matching Pfam model. RefSeq is shown in red AUGUSTUS prediction with "Default" and "Specific" trained models are shown in blue and green, respectively. The numbers refer to the overlapping genes.
We compared these expectations with the number of empirically observed co-occurrences $n(x, y)$. We speak of co-occurrence of domain families or groups $\mathrm{x}$ and $\mathrm{y}$ if $n(x, y)$ $\gg \max \{E(x \mid y), E(y \mid x)\}$ and of avoidance if $n(x, y) \ll$ $\min \{E(x \mid y), E(y \mid x)\}$. The statistical significance of an observed difference between $n(x, y)$ and the values of $\min \{E(x \mid y), E(y \mid x)\}$ and $\max \{E(x \mid y), E(y \mid x)\}$, respectively, is determined observing the fact that the count data $n(x, y)$ follow a Poisson distribution.

Note that the use of the min and max here ensures that the tests for avoidance and co-occurrence are conservative. In order to verify that this test is indeed not prone to detecting false positive associations but rather fails on the side of false negatives, we simulated 30 random data sets by repeatedly permuting the functional annotation of the individual domains using the linux command shuf. No result significant at the $10 \%$ level was returned for both the SUPERFAMILY and Pfam annotations (compared to an expected 3 false positives), reassuring us that the test statistic works conservatively as designed.

\section{RESULTS \& DISCUSSION}

We compare the annotation data and de novo gene prediction at different levels: (1) w.r.t. the set of predicted transcript, w.r.t. the collection of predicted proteins that contain recognizable domains, and (3) w.r.t. to the statistics 
of co-occurring domains in the same annotated protein. We will see in the following, that at each level we observe large discrepancies between the data sets.

\subsection{Ascertainment biases in protein annotations}

In order to get an impression of the differences in coverage of protein annotation we compared the number of transcripts annotated in the genomes of Euarchontoglires, the mammalian clade comprising primates and rodents. These genomes are organized in a very similar way and are expected to have very similar or even nearly identical complements of protein coding genes.

Figure 3 shows these data for RefSeq (version 42) subdivided as numbers of transcripts containing a particular SUPERFAMILY domain. Closely related species, such as the mammals, do not differ much in their gene content. Even if they do, differences tend to be confined to a few gene families with large copy numbers that evolve very quickly, such as olfactory receptors [34]. Even in gene families with rapid losses and gains, the total numbers of family members do not change dramatically at moderate timescales. In contrast to biological wisdom, the numbers of annotated transcript, Fig.3 vary by nearly an order of magnitude between closely related species. Instead of biological differences we clearly observe here only variations in annotation coverage. Indeed, the best-studied model species (Homo sapiens, Macaca mulatta, Mus musculus, and Rattus norvegicus) show much larger transcript numbers than the other, less well studied genomes. The lines are approximately parallel in the logarithmic plot, indicating that the relative abundances of the domains are similar and a simple difference in the completeness of the annotation can account for much of the variation.

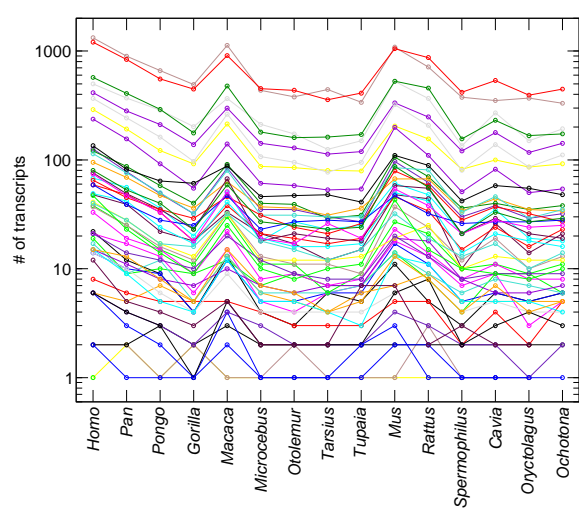

Fig. 3 Differences in RefSeq coverage of primate and rodent genomes. $\mathrm{X}$ axis is the designated primate and rodent genomes, while $\mathrm{Y}$ axis is the total number of annotated transcription factor transcripts. Each line corresponds to number of annotated proteincoding transcripts that contain a particular class of protein domains.

The annotation of RefSeq genomes is complex composite of experimental transcript and protein information and computational procedures including alignments as well as HMM-based ab initio predictions [35] complemented by manual curations. Since an independent gold standard is absent, it cannot be rigorously checked to what extent these annotations are biased. The focus on known protein-coding genes, however, that are used a starting point for the homology-based annotation steps, at least suggests the well-studied protein families are overrepresented. Differences in coverage, thus, may not be the only artefacts that need to accounted for when RefSeq annotation is used for statistical purposes.

As shown in [36] the use of a de novo gene predictor such as GENSCAN can alleviate the differences in coverage and provide quantitatively comparable domain annotations in closely related species. The situation is more complex, however, when a comparison of gene or protein complements for organisms from different kingdoms is required. Additional biases may arise from both the protein and the domain annotation. Homology-based annotation may be confounded by differences in sequence conservation between proteins of different functional classes. Such differences affect not only the overall rate of substitution but also the substitution patterns [37]. The sensitivity of annotation procedure thus may show systematic dependencies on functional classes or the size of gene families. Our knowledge of protein domains is by far not complete. Although most protein domains in well-studied model organisms are evolutionarily very old and rather well conserved, the characteristic patterns of the domains slowly evolve and domain innovation is a relatively infrequent but well-documented phenomenon $[7,8]$. Thus, the sensitivity of the domain annotation procedure must be expected to decrease with increasing evolutionary distance from the examples that have been used to create the domain models. An important technical issue plagues in particular transmembrane regions and signal peptides. These have a hydrophobic bias leading to problematic domain models and subsequently to incorrect function assignments inherited from these domain models [38].

\subsection{Biases in gene predictions}

GENSCAN is a general purpose gene prediction tool that does not admit species specific training. Its internal model implicitly assumes a gene organization similar to that of higher plants and animals. Several lineages of the Eukarya, however, feature gene structures and a genomic organization that is very different from the situation in animals, fungi, or plants. Both Giardia lamblia and Trichomonas vaginalis are extremely intron-poor; Trichomonas vaginalis in addition features very large numbers of paralogs. Kinetoplastids (Trypanosoma and Leishmania) produce large polycistronic transcripts from which individual mature mRNAs are produced by trans-splicing, cis-splicing, and polyadenylation $[39,40]$. Trans-splicing is also prevalent in the nematodes, but absent from most other animal genomes. GENSCAN in particular has problems to detect the gene boundaries in the polycistronic transcripts. Intron-sizes differ dramatically between invertebrates and 
vertebrates, where intron-sizes of more than $10 \mathrm{~kb}$ are not at all uncommon. Another problem is posed by the extreme sequence composition as in the AT-rich genome of Plasmodium falciparum [41].

We therefore employed AUGUSTUS as a trainable gene predictor to test whether differences insensitivity between species due to differences in gene structure might play a large role. A comparison of AUGUSTUS and GENSCAN predictions in Table 1 confirms our suspicion that gene predictors are also a source of biases since the numbers are not only significantly different but also deviate substantially from proportionality. While GENSCAN predicts more twice as many transcripts than AUGUSTUS in Giardia, Homo, and Drosophila, we find the opposite picture in Caenorhabditis, Dictyostellium, Tetrahymena, Plasmodium, Leishmania and Trichomonas.

A comparison of AUGUSTUS predictions with the RefSeq gene inventories agrees rather well in some species, while in others there are substantial difference GENSCAN is a general purpose gene prediction tool that does not admit species specific training. Its internal model implicitly assumes a gene organization similar to that of higher plants and animals. Several lineages of the Eukarya, however, feature gene structures and a genomic organization that is very different from the situation in animals, fungi, or plants. Both Giardia lamblia and Trichomonas vaginalis are extremely intron-poor; Trichomonas vaginalis in addition features very large numbers of paralogs. Kinetoplastids (Trypanosoma and Leishmania) produce large polycistronic transcripts from which individual mature mRNAs are produced by trans-splicing, cis-splicing, and polyadenylation $[39,40]$. Trans-splicing is also prevalent in the nematodes, but absent from most other animal genomes. GENSCAN in particular has problems to detect the gene boundaries in the polycistronic transcripts. Intron-sizes differ dramatically between invertebrates and vertebrates, where intron-sizes of more than $10 \mathrm{~kb}$ are not at all uncommon. Another problem is posed by the extreme sequence composition as inthe AT-rich genome of Plasmodium falciparum [41].

We therefore employed AUGUSTUS as a trainable gene predictor to test whether differences insensitivity between species due to differences in gene structure might play a large role. A comparison of AUGUSTUS and GENSCAN predictions in Table 1 confirms our suspicion that gene predictors are also a source of biases since the numbers are not only significantly different but also deviate substantially from proportionality. While GENSCAN predicts more twice as many transcripts than AUGUSTUS in Giardia, Homo, and Drosophila, we find the opposite picture in Caenorhabditis, Dictyostellium, Tetrahymena, Plasmodium, Leishmania and Trichomonas.

A comparison of AUGUSTUS predictions with the RefSeq gene inventories agrees rather well in some species, while in others there are substantial differences, Fig. 2. Large discrepancies seem to be related to the degree of completeness of the gene annotation. Table 1 shows that GENSCAN predicts more than twice as many genes than
RefSeq in human and Drosophila, while RefSeq has the more inclusive annotation in those species where AUGUSTUS is more sensitive than GENSCAN. Overall, AUGUSTUS conforms better to the RefSeq annotation than GENSCAN. The similarity of the results obtained with RefSeq and AUGUSTUS might be explained in part be the use of similar HMM-based annotation methods in RefSeq [35]. Strictly speaking, therefore, one cannot conclude that AUGUSTUS performs "better" than GENSCAN because its output is more similar to RefSeq, although this is a plausible hypothesis.

\subsection{Gene predictions with domains annotations}

Since we are interested primarily in the distributions of protein domains we also compared RefSeq data with gene predictions restricted to only those genes in which at least one SUPERFAMILY or Pfam domain was annotated. For most species this improves the congruence between the gene sets. In a few cases, however, the differences persist, as in the case of Trypanosoma and human, Fig. 2. In Trypanosoma, most of the difference is explained by annotated RefSeq proteins without recognizable domains. In human, the discrepancy is in part explained by RefSeq isoforms and in part by AUGUSTUS prediction without domains.

Figure 4 shows that significant differences between gene predictions and RefSeq persist even when the data are restricted to predicted transcript with annotated domains. Note, furthermore, that annotation of the same gene predictions with domains from SUPERFAMILY and Pfam also yield substantially different results: in fact, the point clouds show very little correlation.

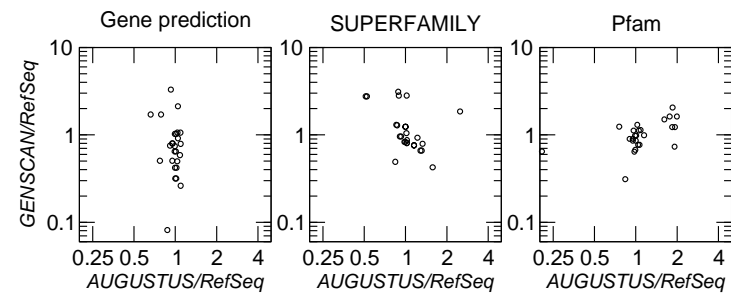

Fig. 4 Discrepancies between de novo gene predictions and RefSeq annotations. In one hand, the $\mathrm{X}$ axis (below) is the quantitative comparison between the total number of AUGUSTUS and RefSeq genes. In the other hand, the $\mathrm{Y}$ axis is the quantitative comparison between the total number of GENSCAN and RefSeq genes. The title of each graphic represents annotated transcript, gene predictions with domains from SUPERFAMILY and Pfam respectively.

Among the predictions with annotated domains, we find e.g. for Leishmania, Tetrahymena, and Plasmodium that both the default and the specific trained gene predictions have a much larger coverage than the RefSeq data. For Trichomonas and Giardia, the situation is reversed. This can probably be explained in part by the large number of 
paralogs and possible pseudogenes included in RefSeq in Trichomonas, but also indicated as lack of sensitivity of the gene predictor for the two parabasalids with their extremely intron-poor genomes. At the domain level, AUGUSTUS and RefSeq agree nearly perfectly e.g. in human and Naegleria. In general, the RefSeq entries missed by the gene predictor are frequently putative pseudogenes and ORFs lacking further annotation. Since the AUGUSTUS 'specific' predictions overall yield the most inclusive data set, these predictions are used in the following section for all statistical analyses of domains compositions.
Surprisingly, we observe very little variation in the number of domains per protein. A significant increase is found in human and fruitfly only. It is unclear, however, whether this a true effect or an artefact arising from a bias in Pfam database. In [42], a difference in the complexity of chromatin proteins between Diplomonads and Dicristates on the one hand, and Alveolates and Stramenopiles on the other hand does exist. Our data do not show such a systematic difference for proteins containing domain classified as "rC: regulator of chromatin" according to GO.

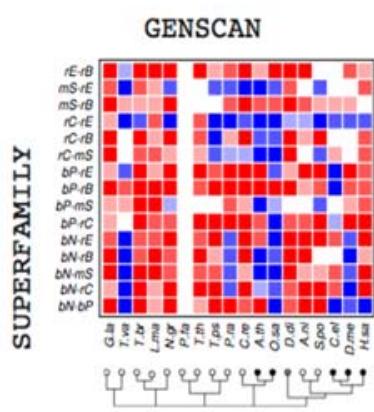

(a)

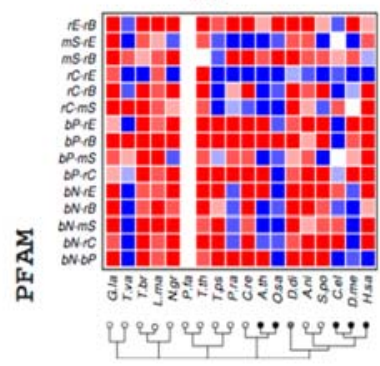

(d)

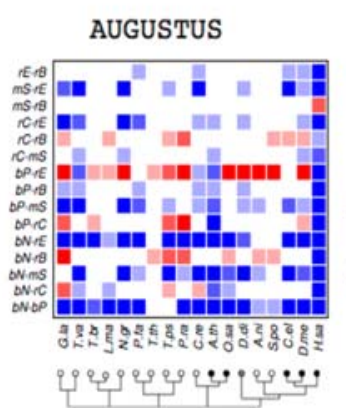

(b)

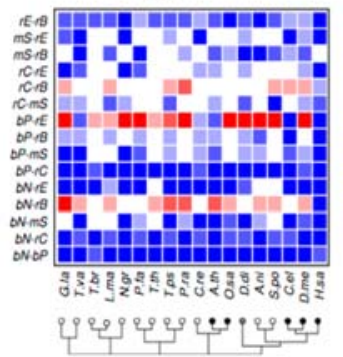

(e)

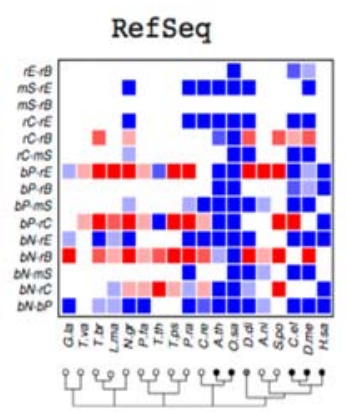

(c)

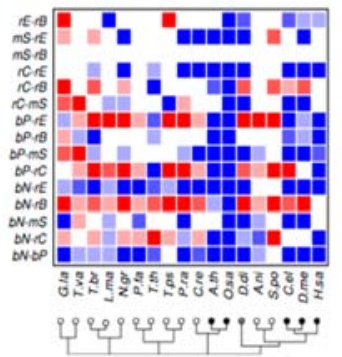

(f)

Fig. 5 Summary of co-occurrences patterns of major functional classes of protein domains across the Eukaryotes. The columns represent the gene prediction (GENSCAN and AUGUSTUS) with the DB-based RefSeq annotations. The rows represents the utilized protein domain databases (PFAM and SUPERFAMILY) for computing the domain co-occurrences, while their global patterns were computed for counting the average co-occurences. Blue rectangles indicate statistically significant avoidance between functional classes of protein domains, red indicates co-occurrence. The saturation of the color denotes the significance levels $p<0.001$ (saturated color), $0.001 \leq p<0.01$ (intermediate), and $0.01 \leq \mathrm{p}<0.1$ (pale). Entries that shown either avoidance or co-occurrence at a significance level of at least $10 \%$ remain white.

\subsection{Correlations in Domain Occurrences}

In [36] we observed regularities in co-occurrences of domains in the same protein. For instance, transcription factors often contain multiple DNA binding domains. In the human genome, these domains predominantly belong to the same (very frequent) domain class such as zinc fingers or winged-helix domains. Combinations of different domain classes, on the other hand, are observed much less frequently than predicted from a random background model. Since most domain families have only a moderate number of occurrences in most genomes this phenomenon of "domain avoidance" cannot be statistically supported for most pairs of domain types even in the large mammalian genomes. In a subsequent study [36] we therefore aggregated domain classes to groups defined by their biochemical function. This aggregation, however, is susceptible to yet another source of biases: different domains types now contribute to the functional classes in the vastly different organism studied here. There is no guarantee, of course, that coverage and quality of functional domain annotations, as provided by the Pfam and SUPERFAMILY databases are uniform across all classes of domains. The phylogenetic distribution of the individual domain families, on the other hand, is of course far from uniform.

A comparison of the panels of Figure 5 shows that the qualitative results on the domain co-occurrence are largely independent of the choice of SUPERFAMILY or 
Pfam as domain database. The main difference is the number of distinct domains in the two databases. Since Pfam is much larger, the absolute domain counts are larger and hence a larger number of the statistical tests reaches the required levels of significance.

On the other hand, there are striking differences between the gene annotation. Based on GENSCAN we predicted a general tendency towards domain co-ocurrences [23]. Using RefSeq annotation, it appears that avoidance dominates (in particular in multicellular animals and plants), while co-ocurrences appear be prevalent in unicellular protozoans. The AUGUSTUS-based gene predictions, however, point at a generic trend towards domain avoidance, with exceptions only for a few pairs of functional classes. The most prominent cases are bN-rB ('binding of nucleic acids' and 'regulation of binding'), bP-rE ('protein binding' and 'regulation of enzymatic activity'), and rC-rB ('regulation of chromatin' and 'regulation of binding'). For $\mathrm{bP}$ and $\mathrm{rE}$ a positive correlation is not unexpected, since regulators of enzymatic activity $(\mathrm{rE})$ can be expected to act by protein-protein binding (bP). The positive correlations between nucleic acid binding domains $(\mathrm{bN})$ and chromatin associated domains (rC) with domains involved in the regulation of binding deserved further investigation. It is consistent with intimate link of both DNA and RNA binding with chromatin regulation reported in [11].

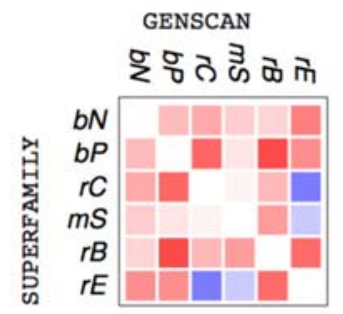

(a)

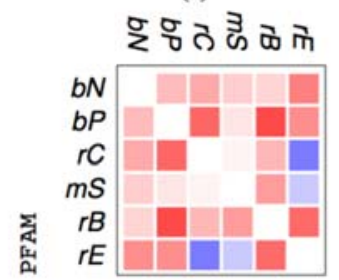

(d)

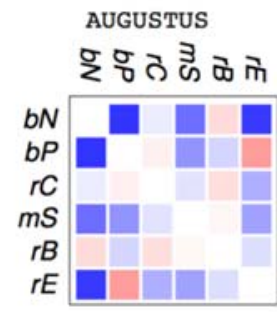

(b)

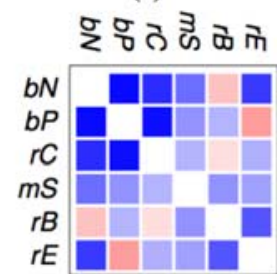

(e)

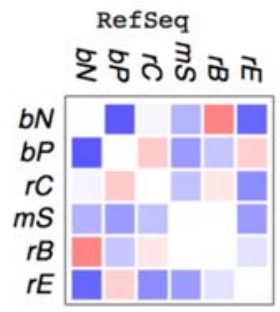

(c)

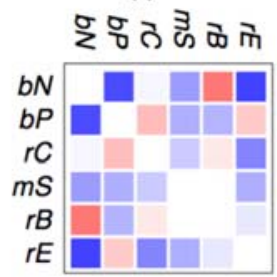

(f)

Fig. 6 Graphical summary of the correlations between the six functional domain classes computed with different protein annotations and domain databases.

In AUGUSTUS annotation, the domain cooccurrences of Tetrahymena in SUPERFAMILY and Pfam are scarce. This could be possibly due to the short scaffold in the Tetrahymena genome that could lead into underestimates [43]. Interestingly, in AUGUSTUS annotation we also observe interesting pattern inhuman genome. The strong co-occurrences of $\mathrm{bN}-\mathrm{rB}, \mathrm{rC}-\mathrm{rB}$, and $\mathrm{bP}-\mathrm{rE}$ that exist in other genomes are missing, and replaced with a strong tendency of avoidance. The possible explanation for the emergence of the avoidance pattern is the complex functionality diversification of the domains that tend not to co-occuring complex organisms. One other noticeable pattern in human that the $\mathrm{mS}$-rB co-occurrence pattern exists in SUPERFAMILY while it disappears completely in Pfam.

Figures 6 further aggregates these data and shows the overall tendency in the correlation between domains of different functional classes. The figure emphasizes the large, qualitative mutual differences between the gene prediction methods and the curated protein annotations. Nevertheless, some consistent patterns emerge, such as the positive correlation between protein binding proteins (bP) and regulators of enzymatic activity $(\mathrm{rE})$. This observation is not surprising although it does not derive from double memberships of domains in both groups. A less obvious signicant co-occurrence is that of nucleic acid binders (bN) with regulators of binding $(\mathrm{rB})$, perhaps hinting a widespread involvement of transcription factors in regulatory processes that involve large protein complexes.

In the multi-cellular organisms with large genomes and large gene families, however, there is a strong signal of avoidance between several functional groups of protein domains. This may be a result of the expansion and diversication of large families of paralogous genes and their use for specific tasks in the regulation of cellular processes. In most cases, we have not been able to trace this effect to excessive duplication events in one or a few gene families, however. The most extreme example in our data is the expansion of the variant-specific surface protein domains. This may be a result of the expansion and diversication of large families of paralogous genes and their use for specific tasks in the regulation of cellular processes. In most cases, we have not been able to trace (VSP) gene in Giardia 
lamblia [44], which fall into the bP (binding of proteins) and $\mathrm{rC}$ (regulation of chromatin) categories and features more than 200 paralogs. Still, the signal for co-occurrence of $\mathrm{rC}$ and $\mathrm{bP}$ is not consistently observed in all combinations gene finding and domain annotation. It appears, therefore, that the observed consistent patterns are not the consequence of an extreme expansion of a single gene family but must be related to the collective effect of gene families with the same functional domain classes.

The most rapidly expanding protein family in human, the KRAB/zinc finger proteins [15], one the other hand, contain only domains annotated as nucleic acid binding, and hence do not contribute to co-occurrence or avoidance patterns. This seems to be the case with many transcription factor families. It is worth noting in this context that in earlier work we observed that this is also a strong pattern of avoidance among different SUPERFAMILY classes of nucleic acid binding domains [36].

\section{CONCLUSION}

Proteins embody a wide variety of functions in a cell, ranging from enzymatic activity to structural scaffolding. The function of a protein is reflected in its domain composition. The range of an organism's biochemical capabilities, both metabolic and regulatory, is thus largely encoded in its protein domain content. Even the presence of RNA-based modes of regulations such as the RNAi pathway are reflected by the associated protein components [45]. Large-scale trends in evolution suchas an increased complexity of transcriptional regulation $[46,47]$ or the diversification of chromatin modification throughout the eukaryotic kingdoms [11] thus can be traced by a quantitative comparison of protein and protein domain complements.

Present-day annotations for most genomes as well as the currently available collection of protein models are far from complete. Quantitative cross-species comparisons thus implicitly rely on the assumption that the available data are a fair, essentially unbiased sample. This is in general not the case. We therefore investigated to what extent de novo gene prediction methods can be used to generate comparable domain distribution data. The large differences between untrained (GENSCAN) and trained (AUGUSTUS) indicates that this not a straight forward endeavour. Using the trained models we obtain results that in general are closer to annotation-based numbers. It is unclear, however, whether this speaks for a better quality of the computational results, or whether this simply reflects that AUGUSTUS has been trained with species-specific gene models using transcript data that also underlie the RefSeq annotation. Furthermore, gene predictors are usually tested and benchmarked against curated annotation such as RefSeq. Thus it may be the case that trained gene predictors and protein annotation databases only share the similar biases. However, it is reasonable to assume that the annotation of protein-codingexons is fairly complete at least in the human genome [48], suggesting that qualitatively similar results from AUGUSTUS and RefSeq are closer to the truth.

The second source of major ascertainment biases in the analysis of large scale evolutionary patterns of functional domains are the protein domain databases themselves. Recent studies reported the innovation of a large number of domain innovation events within both the green plants [49] and the animals [50]. The number of identified clade-specific domains must be expected to depend on the depths in which the clade is studied. The domain inventory is thus probably more complete in animals, fungi, and plants compared to most protozoan lineages.

We find that domain annotation data in particular in less well-annotated genomes may suffer from significant ascertainment biases that are uniform neither across functional classes of proteins nor across phylogeny. Data such as those in Fig. 4 indicate that errors can reach a factor of two (or even larger in extreme cases) in the number of counts cannot be rules out. This is large enough to seriously confound for instance the enrichment analyses ubiquitously used in many genomics publications. Nevertheless the reannotation of domains based on modern gene predictors instead of protein sequence databases appears at least to help reduce the ascertainment bias, although we cannot strictly rule out that manually curated data sets are strongly biased as well.

Large numbers unannotated domains, on the other hand, could further undermine the analysis presented here since the lead to a systematic under-estimation of an organisms metabolic or regulatory capability. Recent reports such as [49] seem to indicate that the innovation of novel domains is prevalent in particular in conjunction with stress response and developmental innovations, and hence a particular functional classes. A more systematic survey of so-far undescribed protein domains thus constitutes a natural next step towards a comprehensive understanding of functional evolution in the eukaryotes. Accurate domain inventories are not only of interest in their own right but also constitute an important source of phylogenetic information [51], in particular in "deep phylogeny" applications. The presence/absence patterns of protein domains were recently used for instance to place the Strepsipter as a sister group of beetles in insect phylogeny [52]. Improved pipelines to estimate the protein domain content directly from genomic data thus have the potential to greatly facilitate phylogenomic investigations.

In the analysis of protein domain combinations at least two sources of biases interact: the dependence of the sensitivity of the protein annotation and an uneven coverage of protein domains that might reflect e.g. different levels of interest in different protein families. The domains distributions are summarized in terms of functional annotations, i.e., GO classes, the uneven interests in different protein families is again a likely source of ascertainment biases. The influence of the same or similar confounding factors in different data sources can conspire 
to produce even qualitatively different results such as the ones observed here for domain co-occurrence data in Fig. 5 .

We conclude that ascertainment biases in current annotation databases as well as computational annotation tools fundamentally limit the accuracy with which domain distributions can be estimated. Statistical significance measures estimated via the commonly employed permutation tests often cannot account for these biases. Hence statistical significance may not sufficient in such cases to make statements about biological reality. Large effects thus will in general be necessary to draw reliable conclusions from domain distribution data unless biases can be ruled out e.g. based on near completeness of data. We showed that at least some of the biases can be reduced by using predictions instead of manual curated annotation. Results show, however, that the prediction has to be chosen carefully so as to avoid introducing new biases.

\section{ACKNOWLEDGEMENT}

We thank Katharina Hoff (Greifswald) for technical assistance with the AUGUSTUS online gene prediction. A.A.P. was supported by the DAAD. LS is supported by the MAGE project, BMBF grant number 50500541.

\section{REFERENCES}

[1] K. Forslund,E.L.L. Sonnhammer, Bioinformatics 24 (2008) 1681.

[2] G. Apic,J. Gough, S.A. Teichmann, J. Mol. Biol. 310 (2001) 311.

[3] C.A. Orengo, J.M. Thornton, Annu. Rev.Biochem. 74 (2005) 867

[4] M.Buljan, A.Bateman, Biochem. Soc. Trans. 37 (2009) 751.

[5] A.D.Moore, A.K. Björklund,D.Ekman, E.Bornberg-Bauer, A.Elofsson, Trends.Biochem. Sci. 33 (2008) 444

[6] E.Koonin, L.Aravind, A.Kondrashov, Cell 101 (2000) 573.

[7] E.Bornberg-Bauer, A.K.Huylmans, T.Sikosek, Curr. Opin. Struct. Biol. 20 (2010) 390

[8] C.M.Zmasek, A.Godzik, Genome Biol. 12 (2011) R4.

[9] K.Mahmood, G.Webb, J.Song, J.Whisstock, A.Konagurthu, Nucleic Acids Res. 40 (2012) e44

[10] K.M.Kim, G.Caetano-Anolles, BMC Evol. Biol. 11 (2011) 140.

[11] S.J.Prohaska, P.F.Stadler, D.C.Krakauer, J. Theor. Biol. 265 (2010) 27.

[12] S.Yang, P.E.Bourne, PLoS ONE 4 (2009) e8378.

[13] W.Stefan, A.Eivind, BMC Evol. Biol., 5 (2005) 24

[14] A.D.Moore, S.Grath, A.Schüler, A.K.Huylmans, E.BornbergBauer, Biochim.Biophys.Acta1834 (2013) 898.

[15] K.Nowick, A.T.Hamilton, H.Zhang, L.Stubbs, Mol.Biol.Evol.27 (2010) 2606.

[16] D.A.de Lima Morais, H.Fang, O.J.Rackham, D.Wilson, R.Pethica, C.Chothia, J.Gough, Nucleic Acids Res. 39 (2011) D427-D434.

[17] C.Burge, S. J.Karlin, Mol.Biol. 268 (1997) 78.

[18] C.B.Burge, S.Karlin,Curr. Opin. Struct. Biol.8 (1998) 346.

[19] I.Korf, BMC Bioinformatics, 5 (2004) 59.

[20] W.Miller, K.D.Makova, A.Nekrutenko, R.C.Hardison, Ann. Rev. Genomics Hum. Genet. 5 (2004) 15

[21] T.Hubbard, D.Barker, E.Birney, G.Cameron, Y.Chen, L.Clark, T.Cox, J.Cuff, V.Curwen, T.Down, R.Durbin, E.Eyras, J.Gilbert, M.Hammond, L.Huminiecki, A.Kasprzyk, H.Lehvaslaiho, P.Lijnzaad, C.Melsopp, E.Mongin, R.Pettett, M.Pocock, S.Potter, ,A.RustE.Schmidt, S.Searle, G.Slater, J.Smith, W.Spooner, A.Stabenau, J.Stalker, E.Stupka, A.Ureta-Vidal, I.Vastrik, M.Clamp, Nucleic AcidsRes. 30 (2002) 38
[22] H.Korenaga, T.Kono, M.Sakai, Cytokine 59 (2012) 541.

[23] A.A.Parikesit, P.F.Stadler, S.J.Prohaska, Genes 2 (2011) 912

[24] M.Stanke,S.Waack, Bioinformatics 19 (2003) ii215-ii225.

[25] M.Stanke,O.Schöffmann,B.Morgenstern,S.Waack,BMC Bioinformatics 7 (2006) 62.

[26] M.Stanke, M.Diekhans, R.Baertsch, D.Haussler,Bioinformatics, 24 (2008) 637.

[27] M.Stanke, Augustus Training Manual: http://bioinf.unigreifswald.-de/augustus/binaries/tutorial/training.html. ioinformatics Group, University of Greifswald. (2011).

[28] A.Quinlan, I. Hall, Bioinformatics 26 (2010) 841.

[29] S.L.Baldauf, J. Syst. Evol.46 (2008) 263

[30] B.Dilts, United States Patent Application Publicatin No: US 2012/0081389 (LucidChart) (2012), Technical report toLucidChart LLC.

[31] S.R. Eddy, PLoS Comp. Biol. 7 (2011) e1002195.

[32] The Gene Ontology Consortium. Nat Genet. 25 (2000) 25.

[33] M.Punta, P.C.Coggill, R.Y.Eberhardt, J.Mistry, J.Tate; C.Boursnell, N.Pang, K.Forslund, G.Ceric, J.Clements, A.Heger, L.Holm, E.L.Sonnhammer, S.R.Eddy, A.Bateman,R.D.Finn, Nucleic Acids Res. 40 (2012) D290-D301.

[34] Y.Niimura, M.Nei,PLoS ONE 2 (2007) e708.

[35] K.D.Pruitt, T.Tatusova, D.R.Maglott, Nucleic Acids Res. 33 (2005) D501-D504.

[36] A.A.Parikesit, P.F.Stadler, S.J.Prohaska, German Conference on Bioinformatics 2010,D.Schomburg, A.Grote,Eds. Gesellschaft fürInformatik: Bonn, 2010. Vol. P-173, Lecture Notes in Informatics, pp.93-102.

[37] G.C. Conant, G.P.Wagner, P.F.Stadler, Mol. Phylog. Evol. 42 (2007) 298.

[38] W.C. Wong, S.Maurer-Stroh, F.Eisenhaber, PLoSComput. Biol. 6 (2010) e1000867.

[39] S.Michaeli, Future Microbiol. 6 (2011) 459

[40] S.Thomas, A.Green, N.R.Sturm, D.A.Campbell, P.J.Myler,BMC Genomics 10 (2009) 152.

[41] F. Lu, H.Jiang, J.Ding, J.Mu, J.G.Valenzuela, J.M.C.Ribeiro, X.z.Su,BMCGenomics 8 (2007) 255.

[42] L.M.Iyer, V.Anantharaman, M.Y.Wolf, L.Aravind, Int. J.Parasitol. 38 (2008) 1.

[43] JEisen, et al. PLoS Biol. 4 (2006) e286.

[44] R.D. Adam,A.Nigam, V.Seshadri, C.A.Martens, G.A.Farneth, H.G.Morrison, T.E.Nash, S.F.Porcella, R.Patel,BMC Genomics $11(2010) 424$.

[45] I.A.Drinnenberg, D.E.Weinberg, K.T.Xie, J.P.Mower, K.H.Wolfe, G.R.Fink, D.P.Bartel, Science 326 (2009) 544.

[46] R.Melzer, G.Theissen, MethodsMol. Biol. 754 (2011) 3.

[47] E.Shelest, FEMSMicrobiol.Lett. 286 (2008) 145

[48] B.Banfai, H.Jia, J.Khatun, E.Wood,B.Risk, W.E.GundlingJr., A.Kundaje,H.P.Gunawardena, Y.Yu, L.Xie, K.Krajewski, B.D.Strahl, X.Chen, P.Bickel, M.C.Giddings, J.B.Brown, L.Lipovich, Genome Res. 22 (2012) 1646.

[49] A.R.Kersting, E.Bornberg-Bauer,A.D.Moore, S.Grath, Genome Biol.Evol. 4 (2012) 316.

[50] A.D. Moore, E.Bornberg-Bauer, Mol. Biol.Evol. 29 (2012) 787.

[51] S.Yang, R.F.Doolittle, P.E.Bourne, Proc.Natl. Acad. Sci. USA 102 (2005) 373.

[52] O.Niehuis, G.H.Hartig, S.Garth, H.Pohl,J.Lehmann, H.Tafer, A.Donath, V.Krauss, C.Eisenhardt, J.Hertel, M.Petersen, C.Mayer, K.Meusemann, R.S.Peters, P.F.Stadler,R.G.Beutel, E.BornbergBauer, D.D.McKenna, B.Misof,Current Biol. 22 (2012)1309.

[53] A.A. Parikesit, Supplemental Data: http://www.bioinf.uni-leipzig.de/supplements/12-007. (2012) Bioinformatics groups, University of Leipzig. 